\title{
On the Iwasawa $\lambda$-invariants of quaternion extensions
}

\author{
by \\ KeirCHi Komatsu (Tokyo) \\ Dedicated to the memory of \\ Prof. Dr. Jürgen Neukirch
}

For a prime number $l$ and a number field $k$, denote by $\lambda_{l}(k)$ the Iwasawa $\lambda$-invariant associated with the ideal class group of the cyclotomic $\mathbb{Z}_{l}$-extension $k_{\infty}(l)$ over $k$. It is conjectured that this invariant is zero for any prime $l$ and any totally real number field $k$ (cf. [7]). Several authors have given some sufficient conditions for the conjecture when $k$ is a real abelian field (cf. [1]-[9]). Using them, many examples of the vanishing of $\lambda$-invariants for real abelian number fields are given. However, it seems that an example of a totally real non-abelian field has not yet been given. In this paper we give quaternion extensions $K$ over the rational number field $\mathbb{Q}$ with $\lambda_{2}(K)=0$. A Galois extension $K$ over $\mathbb{Q}$ is called a quaternion extension if the Galois group $G(K / \mathbb{Q})$ of $K$ over $\mathbb{Q}$ is isomorphic to the quaternion group $H_{8}$ of order 8 . The quaternion group $H_{8}$ is a group $H_{8}=\langle\sigma, \tau\rangle$ of order 8 with $\sigma^{4}=1, \sigma^{2}=\tau^{2}$ and $\tau \sigma \tau^{-1}=\sigma^{-1}$.

The main purpose of this paper is to prove the following:

THEOREM. Let $p$ be a prime number with $p \equiv 3(\bmod 8), k=\mathbb{Q}(\sqrt{2}, \sqrt{p})$ and $k_{\infty}(2)$ the cyclotomic $\mathbb{Z}_{2}$-extension of $k$. Then there exist natural numbers $x, y$ with $x^{2}-y^{2} p=2 p$. Let $K_{\infty}(2)$ be the cyclotomic $\mathbb{Z}_{2}$-extension of $K=k(\sqrt{(x+y \sqrt{p})(2+\sqrt{2})})$. Then the Galois group $G(K / \mathbb{Q})$ of $K$ over $\mathbb{Q}$ is isomorphic to the quaternion group $H_{8}$ and the $\lambda$-invariant $\lambda\left(K_{\infty}(2) / K\right)$ of $K_{\infty}(2)$ over $K$ vanishes.

First we recall the following lemma which plays an important role in our proof of this theorem:

Lemma (cf. [2]). Let $l$ be a prime number, $k$ a totally real number field of finite degree and $K$ a real cyclic extension of degree $l$ over $k$. Assume that

1991 Mathematics Subject Classification: Primary 11R23. 
$k_{\infty}(l)$ has only one prime ideal lying over $l$ and that the class number $h_{k}$ of $k$ is not divisible by $l$. Then the following are equivalent:

(1) $\lambda\left(K_{\infty}(l) / K\right)=0$.

(2) For any prime ideal $w$ of $K_{\infty}(l)$ which is prime to $l$ and ramified in $K_{\infty}(l) / k_{\infty}(l)$, the order of the ideal class of $w$ is prime to $l$.

Pro of (of Theorem). Since $p \equiv 3(\bmod 8)$, we have $N_{\mathbb{Q}(\sqrt{p}) / \mathbb{Q}}\left(\mathbb{Q}(\sqrt{p})^{\times}\right)$ $\not \supset-1$. Hence the cardinality of the ambiguous classes of $\mathbb{Q}(\sqrt{p})$ is equal to one, which shows that a prime ideal of $\mathbb{Q}(\sqrt{p})$ lying above 2 is principal. Therefore there exist integers $x, y$ with $x^{2}-p y^{2}=2 p$ by $\left(\frac{-2}{p}\right)=1$. We put $\alpha=\sqrt{(2+\sqrt{2})(x+y \sqrt{p})}$. Now, let $\sigma, \tau$ be elements of the Galois group $G(k / \mathbb{Q})$ with $\sqrt{2}^{\sigma}=-\sqrt{2}, \sqrt{p}^{\sigma}=\sqrt{p}, \sqrt{2}^{\tau}=\sqrt{2}$ and $\sqrt{p}^{\tau}=-\sqrt{p}$. Then we have $\left(\alpha^{2}\right)\left(\alpha^{2}\right)^{\sigma}=2(x+y \sqrt{p})^{2}$ and $\left(\alpha^{2}\right)\left(\alpha^{2}\right)^{\tau}=2 p(2+\sqrt{2})^{2}$, which shows that $K$ is a Galois extension over $\mathbb{Q}$. For simplicity, we denote by $\sigma, \tau$ extensions of $\sigma, \tau$ to $K$ with $\alpha^{\sigma}=\sqrt{2} \alpha^{-1}(x+y \sqrt{p})$ and $\alpha^{\tau}=\sqrt{2 p} \alpha^{-1}(2+$ $\sqrt{2})$. Then we can easily see $G(K / \mathbb{Q})=\langle\sigma, \tau\rangle, \sigma^{4}=1, \sigma^{2}=\tau^{2}$ and $\tau \sigma \tau^{-1}=$ $\sigma^{-1}$. Hence $G(K / \mathbb{Q})$ is isomorphic to $H_{8}$.

Now, we prove $\lambda\left(K_{\infty}(2) / K\right)=0$. First we notice that the class number $h_{\mathbb{Q}(\sqrt{p})}$ is not divisible by 2 . Therefore $h_{k}$ is not divisible by 2 , since 2 is fully ramified in $k$ over $\mathbb{Q}$ and since $p$ is unramified in $k$ over $\mathbb{Q}(\sqrt{p})$. One should also remark that the infinite primes are unramified. Let $\mathfrak{P}_{p}$ be a prime ideal of $K$ lying above $p, \mathfrak{p}_{2}$ a prime ideal of $k$ lying above 2 and $\mathfrak{p}_{p}$ a prime ideal of $k$ lying above $p$. Then we can see $((x+y \sqrt{p})(2+\sqrt{2}))=\mathfrak{p}_{p}^{2} \mathfrak{p}_{2}^{2}$. Hence we have $(\alpha)=\mathfrak{P}_{p}(2+\sqrt{2})$ in $K$. This shows that $\mathfrak{P}_{p}$ is a principal ideal of $K$. Therefore $\lambda\left(K_{\infty}(2) / K\right)=0$ follows from the Lemma or [7, Lemma 3]. This completes our proof.

REMARK. Since there exist infinitely many prime numbers $p$ with $p \equiv$ $3(\bmod 8)$ which are unramified in $k / \mathbb{Q}(\sqrt{p})$, there exist infinitely many quaternion extensions $K$ with $\lambda\left(K_{\infty}(2) / K\right)=0$.

\section{References}

[1] T. Fukuda and K. Komatsu, On $\mathbb{Z}_{p}$-extensions of real quadratic fields, J. Math. Soc. Japan 38 (1986), 95-102.

[2] T. Fukuda, K. Komatsu, M. Ozaki and H. Taya, Iwasawa $\lambda_{p}$-invariants of relative cyclic extensions of degree p, Tokyo J. Math. 20 (1997), 475-480.

[3] T. Fukuda and H. Taya, The Iwasawa $\lambda$-invariants of $\mathbb{Z}_{p}$-extensions of real quadratic fields, Acta Arith. 69 (1995), 277-292.

[4] R. Greenberg, On the Iwasawa invariants of totally real number fields, Amer. J. Math. 98 (1976), 263-284. 
[5] H. Ichimura and H. Sumida, On the Iwasawa $\lambda$-invariants of certain real abelian fields, to appear.

[6] - , - On the Iwasawa $\lambda$-invariants of certain real abelian fields II, to appear.

[7] K. Iw as aw a, A note on capitulation problem for number fields II, Proc. Japan Acad. 65 (1989), 183-186.

[8] J. S. Kraft and R. Schoof, Computing Iwasawa modules of real quadratic number fields, Compositio Math. 97 (1995), 135-155.

[9] M. Ozaki and H. Taya, On the Iwasawa $\lambda_{2}$-invariants of certain families of real quadratic fields, submitted for publication (1996).

Department of Information and Computer Science

School of Science and Engineering

Wasada University

3-4-1 Okubo, Shinjuku, Tokyo 169-8555

Japan

E-mail: kkomatsu@mse.waseda.ac.jp 